\title{
FIB Preparation of Bone-Implant Interfaces for Correlative On-Axis Rotation Electron Tomography and Atom Probe Tomography.
}

\author{
Julia Huang ${ }^{1}$, Xiaoyue Wang ${ }^{2}$ and Kathryn Grandfield ${ }^{2}$ \\ 1. Canadian Centre for Electron Microscopy, Brockhouse Institute for Materials Research, McMaster \\ University, Hamilton, Canada. \\ 2. Department of Materials Science and Engineering, McMaster University, Hamilton, Canada.
}

Osseointegration refers to the direct bonding between titanium implants and bone, making it one of the key factors for the success of reconstructive and regenerative orthopaedic and dental surgeries [1]. Resolving the implant-bone interface with high chemical or spatial resolution enables a clearer determination of bone growth mechanisms at the implant interface and optimal modification of implant surfaces. Due to the inhomogeneity of bone tissue, projection-based imaging such as HAADF-STEM imaging often leads to misinformation at the region of highest interest, the interface.

Recently, Z-contrast electron tomography has been applied to enable visualization of these implant-bone interfaces of interest in three-dimensions. Reconstructions of the interface between bone and hydroxyapatite, titanium and titanium alloys have been performed on conventional lamellae-type FIB sections [2]. However, there are some limitations to using conventional lamellae for tomography. Firstly, it is well known that the "missing wedge", resulting from geometrical limitations in the TEM, leads to artefacts and elongation in the final reconstruction [3]. Secondly, the ability to combine tiltseries with spectral information (e.g., electron energy loss spectroscopy (EELS)) is severely limited with increasing tilt angle due to increasing specimen thickness.

On-axis rotation tomography, enabling a complete $360^{\circ}$ sample rotation, is a viable alternative acquisition scheme to circumvent the issues arising from the missing wedge. Furthermore, the rodshaped sample geometry provides a means to easily correlate electron tomography to atom probe tomography with the same specimen.

Contact between bone and implant can often be sporadic due to the high frequency of cracking at the interface during implant extraction and embedding protocols. Therefore, it is essential to view the implant-bone interface in cross-section to identify regions of contact. Furthermore, to enable correlative atom probe tomography, it is assumed the preferred sample orientation would consist of bone at the tip of the rod, and metallic implant at the base. FIB is therefore the optimal preparation technique for this precise sample preparation, and our method is explained below.

In this work, a dual-beam FIB instrument (Zeiss NVision 40, Carl Zeiss AG) equipped with a 30kV gallium ion column, FEG SEM, carbon and tungsten gas injector system (GIS), and Kleindiek micromanipulator (Kleindiek Nanotechnik $\mathrm{GmbH}$ ) was employed. Titanium and bone implants are generally embedded in plastic and sliced longitudinally as shown in Figure 1. This is the starting point for FIB preparation. Sample posts $(1.8 \mathrm{~mm}$ in diameter) from the Fischione Model 2050 On-Axis Rotation Tomography Holder were mounted on a specialized stub with two pins located $90^{\circ}$ apart to enable rotation of the stub. The FIB preparation protocol followed the schematic in Figure 2: 1) the region of interest was selected, electron and ion beam induced tungsten deposition protected a section 2 x $10 \mu \mathrm{m}$ prior to lift-out, 2) the section was attached by the titanium end to the top of sample post, 3) the 
specialized stub was rotated $90^{\circ}$ so that the sample post with attached specimen was oriented vertically with bone on top, 4) a thin layer of tungsten was deposited on the top of the bone surface prior to annular milling, 5) the specimen is annularly milled using successively lower current to a final diameter of approximately $200 \mathrm{~nm}$. A final low $\mathrm{keV}$ polishing step was performed to reduce surface damage.

The precision of the FIB instrument and our specialized stub geometry enabled us to 1) locate the sitespecific bone-implant interface of interest, and 2) prepare an intact rod-shaped specimen, oriented such that bone is at the top of the pillar. Future works will include utilizing the specimen produced herein for on-axis rotation tomography and correlated atom probe tomography [4].

References:

[1] P-I Brånemark et al, Scand J Plast Reconstr Surg 3 (1969), p. 81.

[2] K Grandfield, A Palmquist and H Engqvist, Philos Trans R Soc A 370 (2012), p. 1337.

[3] PA Midgley and RE Dunin-Borkowski, Nature Materials 8 (2009), p. 271.

[4] The electron and ion microscopy work described in this paper was performed at the Canadian Centre for Electron Microscopy at McMaster University; a facility supported by NSERC and other government agencies.

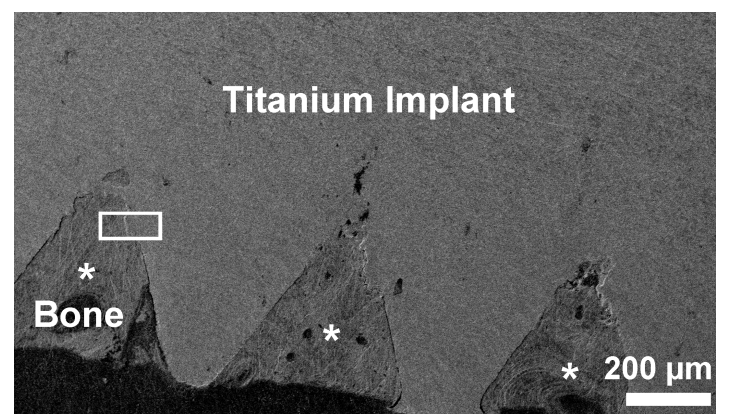

Figure 1. SEM image of the titanium implant and surrounding bone (marked by *) sectioned longitudinally. Rod-shaped sample preparation was carried out at the boxed region.

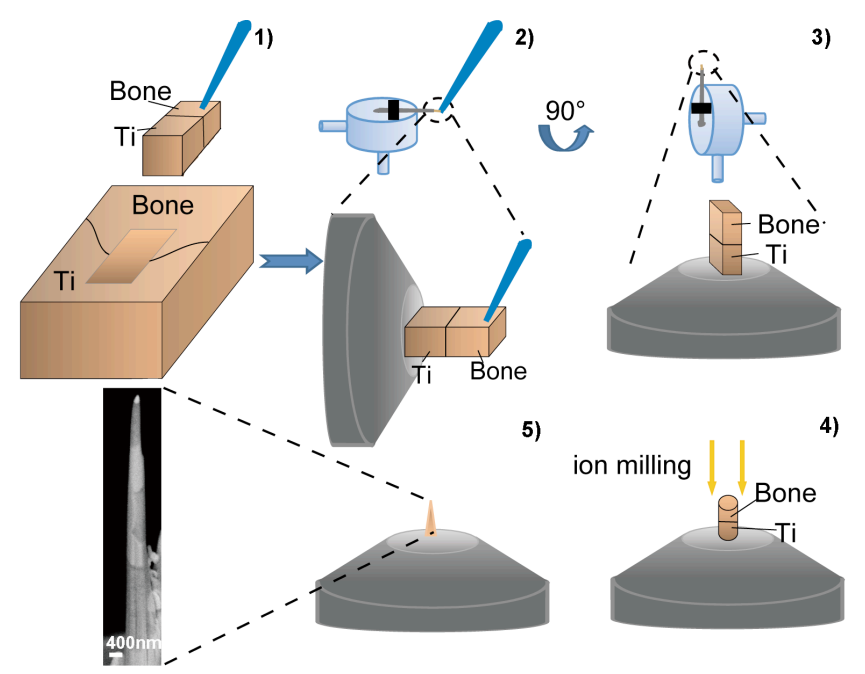

Figure 2. Schematic representation of the lift-out, mounting, rotation and annular milling protocol used to create the rod-shaped specimen containing the bone-implant interface on the tip of a $1.8 \mathrm{~mm}$ sample post for the rotation tomography holder and atom probe tomography. 